Progressive changes in a recognition memory network in Parkinson's disease.

Bàrbara Segura ${ }^{3}$, Naroa Ibarretxe-Bilbao ${ }^{1,2,3,6}$, Roser Sala-Llonch ${ }^{3}$, Hugo Cesar Baggio ${ }^{3}$, Maria Josep Martí ${ }^{1,2,4}$, Francesc Valldeoriola ${ }^{1,2,4}$, Pere Vendrell ${ }^{2,3}$, Nuria Bargalló ${ }^{5}$, Eduard Tolosa 1,2,4 , Carme Junqué ${ }^{1,2,3}$.

${ }^{1}$ Centro de Investigación en Red de Enfermedades Neurodegenerativas (CIBERNED), Hospital Clínic de Barcelona. Barcelona. Spain.

${ }^{2}$ Institute of Biomedical Research August Pi i Sunyer (IDIBAPS). Barcelona, Spain.

${ }^{3}$ Department of Psychiatry and Clinical Psychobiology, University of Barcelona. Barcelona. Spain.

${ }^{4}$ Parkinson's Disease and Movement Disorders Unit, Neurology Service, Institut Clínic de Neurociències (ICN), Hospital Clínic de Barcelona. Barcelona, Spain.

${ }^{5}$ Centre de Diagnòstic per la Imatge Hospital Clinic de Barcelona (CDIC), Hospital Clínic de Barcelona, Spain.

${ }^{6}$ Department of Methods and Experimental Psychology, Faculty of Psychology and Education, University of Deusto. Bilbao, Spain.

Corresponding author:

Prof. Carme Junque

Department of Psychiatry and Clinical Psychobiology. University of Barcelona

Casanova 143 (08036) Barcelona, Spain

Phone: (+34) 934024570 // Fax: (+34) 934035294 // E-mail: cjunque@ub.edu

Word count: 4217 


\section{Number of references: 44}

Keywords: functional MRI, memory, Parkinson's disease, functional connectivity, follow-up

\section{ABSTRACT}

In a previous functional MRI (fMRI) study, we found that Parkinson's disease (PD) patients presented dysfunctions in the recruitment of recognition memory networks. We aimed to investigate the changes in these networks over time.

We studied 17 PD patients and 13 age and sex-matched healthy subjects. In both groups fMRI (recognition memory paradigm) and neuropsychological assessments were obtained at baseline and follow-up. In order to analyze changes over time in functional networks, modelfree (independent component analysis) analyses of the fMRI data were carried out. After that, a cross-correlation approach was used to assess the changes in the strength of functional connectivity.

At follow-up, patients showed reduced recruitment of one network, including decreased activations in orbitofrontal cortices, middle frontal gyri, frontal poles, anterior paracingulate cortex, superior parietal lobes and left middle temporal gyrus, as well as decreased deactivation in anterior paracingulate gyrus and precuneus. Cross-correlation analyses over time showed a decrease in the strength of functional connectivity between middle frontal gyrus and superior parietal lobe in PD patients.

Model-free fMRI and cross-correlation connectivity analyses were able to detect progressive changes in functional networks involved in recognition memory in PD patients at early disease stages and without overt clinical deterioration. Functional connectivity analyses could be useful to monitor changes in brain networks underlying neuropsychological deficits in PD. 


\section{INTRODUCTION}

Cognitive dysfunctions occur at the early stages of Parkinson's disease (PD) and most frequently involve impairments of memory, executive and visuoperceptual functions $(1,2)$. Regarding memory dysfunctions, learning and delayed recall are known to be impaired in early disease stages. Recognition memory is not usually impaired at these stages, although it is in patients who fulfill diagnostic criteria for dementia. In addition, recognition memory dysfunctions have been related to the level of difficulty of the task $(3,4)$

Functional connectivity refers to temporal correlations (concurrent activity) of spatially remote neurophysiological events. Functional MRI (fMRI) data can be analyzed with model-free fMRI approaches in order to obtain whole-brain patterns of functional connectivity, as well as with cross-correlation methods to quantify connectivity strength between two predefined brain regions called seeds. While the first approach can be used as an exploratory method without a priori knowledge of the functional pattern, the second needs prior hypotheses regarding the brain regions that are considered to be connected (5).

Tensorial probabilistic independent component analysis (T-PICA) is a model-free approach to analyze fMRI data. This method identifies patterns of coherent blood-oxygen-level-dependent signal fluctuations across all voxels in the brain and groups such patterns into components in the spatial, temporal and subject domains (6). Distinct functional networks made up of different brain areas can be characterized as distinct components through this technique. Studies using model-free analyses have helped detect subtle progressive changes in brain function in other degenerative diseases, such as Alzheimer's disease (7).

In a previous study, we used T-PICA to detect alterations in the functional cerebral network involved in recognition memory (8). Compared with controls, PD patients showed a decreased 
task-related activation in areas involved in this network. We also observed decreased taskrelated deactivations in the default mode network (DMN), a group of spatially segregated brain structures which are more active during tasks that direct attention away from external stimuli or during the resting state, with functions related either to internal mentation or to the exploratory monitoring of the external environment when focused attention is reduced (9). These results indicated that functional brain changes related to memory processes can occur prior to overt recognition memory deficits in PD patients.

In PD, different connectivity analysis methods have demonstrated abnormal patterns of interaction within brain networks involved in motor performance (10-13), cognitive tasks such attention to action (14) and card-sorting tasks (15), or in the resting state $(16,17)$, but the deterioration over time of these connectivity patterns has not been investigated.

A longitudinal $\left[{ }^{18} \mathrm{~F}\right]$-fluorodeoxyglucose positron emission tomography (FDG PET) study reported a progressive metabolic activity decline in medial prefrontal and parietal associative regions (18), which sustain cognitive functions impaired in PD (19). However, to the best of our knowledge, specific recognition memory network deterioration and loss of functional connectivity strength between its specific areas has not been studied.

The two main aims of this study were [1] to investigate how the deterioration of recognition memory network activation patterns progresses using a model free approach, and [2] to study the possible loss of functional connectivity strength between the main regions of this network over time.

\section{METHODS}

\subsection{Participants}


Patients were recruited from an outpatient Movement Disorders Clinical Neurology Service, Hospital Clinic i Provincial de Barcelona, in collaboration with the Department of Psychiatry and Psychobiology (University of Barcelona). Healthy controls were volunteers matched by age, gender and years of education with patients. All subjects were right-handed. The study subjects were part of a previously studied sample (20), and were subsequently invited by telephone to participate in a follow-up evaluation (average period of 35.50 months, $S D=1.85$, range $=31-40$ months). At baseline, 24 early-PD patients and 24 healthy controls participated in the study. In the follow-up assessment, 17 PD patients and 13 healthy controls agreed to participate (table 1).

Fourteen PD patients at baseline and all 17 patients at follow-up were taking antiparkinsonian drugs, consisting of different combinations of levodopa, levodopa with COMT inhibitors, MAO inhibitors, dopamine agonists and amantadine. All assessments were made while patients were in the on state. Levodopa equivalent daily doses (LEDD) were calculated as proposed by Tomlinson et al. (21). Six PD patients at baseline and 4 patients at follow-up were taking antidepressant drugs (see supplementary materials 1).

Table 1. Sociodemographical data of participants at baseline.

\begin{tabular}{|l|l|l|l|l|}
\hline & PD $(\mathrm{n}=17)$ & Controls $(\mathrm{n}=13)$ & Test stats & $p$ \\
\hline Age & $59.59 \pm 8.29$ & $57.15 \pm 10.57$ & $0.17^{\mathrm{a}}$ & 0.87 \\
\hline Gender (male/female) & $13 / 4$ & $10 / 3$ & $0.001^{\mathrm{b}}$ & 0.98 \\
\hline Education (years) & $10.82 \pm 4.76$ & $12.31 \pm 3.35$ & $0.96^{\mathrm{a}}$ & 0.35 \\
\hline Age at onset & & & & \\
\hline Disease duration§ & $53.68 \pm 8.50$ & - & - & - \\
\hline & & & & - \\
\hline
\end{tabular}


Values are mean \pm standard deviation. PD: Parkinson's disease.

$\S$ duration of motor symptoms, in years.

Test stats: Student's t-test $t(\mathrm{a})$ or chi-square test $\chi(\mathrm{b})$ statistics.

From the initial sample, two patients died, two moved to another province, and three patients declined to participate. In the healthy control group, two subjects died, five subjects declined to participate and two couldn't be contacted. Two control subjects were excluded from the final analysis because of movement artifacts in the fMRI acquisition.

No significant differences were observed in baseline clinical and sociodemographical characteristics between the total initial sample and the final sample included in the longitudinal assessment, either for controls (age, sex, education and MMSE, BDI or NPI scores) or for PD patients (age, sex, education, disease duration, age at onset, LEDD, and MMSE, BDI, NPI, UPDRS or Hoehn \& Yahr scores) (see supplementary materials 1).

The inclusion criteria for participating in the study at baseline were: i) Fulfillment of the UK PD Society Brain Bank (PDSBB) diagnostic criteria for PD (22); ii) Hoehn and Yahr stage $\leq$ II; iii) disease duration $\leq 5$ years. Exclusion criteria for all subjects were: $i)$ the presence of dementia as diagnosed by a neurologist according to the Movement Disorder Society diagnostic criteria for Parkinson's disease dementia (23): ii) the presence of other neurological or psychiatric disorders such as depression, which was evaluated by means of the Beck's Depression Inventory-II (BDI) (24); and iii) the presence of visual hallucinations assessed by the Neuropsychiatric Inventory Questionnaire (NPI-Q) (25).

The study was approved by the institutional ethics committee and all enrolled subjects gave written informed consent prior to taking part in the study.

\subsection{Neuropsychological assessment}


All participants underwent a comprehensive neuropsychological examination performed by a trained neuropsychologist (N.I-B). The neuropsychologist in charge of the assessment was the same at baseline and follow-up examinations. Verbal memory assessment was made with Rey's Auditory Verbal Learning Test (RAVLT) (26). The RAVLT variables computed were total learning, delayed memory recall after 20 minutes and recognition.

Additionally, other neuropsychological domains known to be impaired early in the course of Parkinson's disease were assessed using the forward digit span and backward digit span from the Wechsler Adults Intelligence Scale (WAIS-III) to measure working memory, and verbal fluency tests, including assessment of phonemic (total of words starting with " $p$ " in $1 \mathrm{~min}$ ) and semantic fluencies (number of animals in $1 \mathrm{~min}$ ). The details of the neuropsychological battery used are described elsewhere (8).

Statistical analyses of neuropsychological and clinical variables were performed using the statistical package PASW-18 (SPSS, Inc., 2009, Chicago, IL, www.spss.com). A general linear mixed model (GLM) for repeated measures was used to test whether variables changed in each group across time.

\section{$2.3 \mathrm{fMRI}$ acquisition}

Data was acquired with a 3-Tesla MAGNETOM Tim Trio scanner (Siemens, Germany), using a multi-slice gradient-echo echo-planar imaging (EPI) functional sequence with the following parameters: repetition time $(T R)=2000 \mathrm{~ms}$; echo time $(T E)=30 \mathrm{~ms} ; 36 \times 3 \mathrm{~mm}$ axial slices providing whole-brain coverage. A T1-weighted structural image was also acquired for each subject with MPRAGE 3D protocol $(\mathrm{TR}=2300 \mathrm{~ms} ; \mathrm{TE}=2.98 \mathrm{~ms}$; inversion time $=900 \mathrm{~ms} ; \mathrm{FOV}$ : $256 \times 256 \mathrm{~mm} ; 1 \mathrm{~mm}$ isotropic voxel).

2.4 Recognition memory fMRI paradigm 
We used a recognition memory fMRI paradigm the experimental design of which has been previously described in detail (8).

In brief, before the image acquisition, participants viewed a list of 35 words that they should try to remember. Afterwards, during scanning, subjects were asked to recognize the previously learned words from a list of 70 words. The experiment consisted of a 20-block design, with 2 conditions: recognition memory or control. In the recognition memory condition, participants had to recognize the previously learned words, whereas in the control condition they had to detect the concatenation of letters "AAAAAAA" from other possible combinations.

\section{$2.5 \mathrm{fMRI}$ data analysis}

\subsubsection{Pre-processing of fMRI data}

The following data pre-processing algorithm was carried out on the fMRI data set using FSL tools (FMRIB's Software Library, http://www.fmrib.ox.ac.uk/fsl): motion correction using MCFLIRT (27), removal of non-brain structures from the EPI volumes using BET (28), spatial smoothing using a Gaussian kernel of $5 \mathrm{~mm}$ FWHM, mean-based intensity normalization of all volumes by the same factor (4D grand demean), high-pass temporal filtering (FWHM = $100 \mathrm{~s}$ ) and Gaussian low-pass temporal filtering $(F W H M=11.2 \mathrm{~s})$. The functional scans were registered to the MNI152 standard space through affine registration with FLIRT (29).

\subsubsection{Model-based analysis}

After pre-processing the data, model-based fMRI data analysis was carried out using FEAT (FMRI Expert Analysis Tool) Version 5.98, part of FSL. At the individual level, we obtained an activation map for each subject (recognition task > control task). Higher-level analysis was then carried out using FLAME (FMRIB's Local Analysis of Mixed Effects) (30, 31), in order to explore between-group differences and to obtain global average (one-sample t-test) activation maps (patients and controls). Z (Gaussianized T/F) statistic images were thresholded using $Z>2.3$ 
and a corrected cluster significance threshold of $p \leq 0.05$ using Gaussian random field theory to define each cluster's estimated significance level.

\subsubsection{Independent component analysis}

The pre-processing streamline was the same used in the model-based analysis. After it, analysis of the task-related fMRI images was carried out using Tensorial Independent Component Analysis (TICA) as implemented in the Multivariate Exploratory Linear Decomposition into Independent Components tool (MELODIC Version 3.05) (6), part of FSL. MELODIC allows $\mathrm{fMRI}$ data to be decomposed into three-dimensional sets of vectors, also named independent components (IC), which describe signal variations across the temporal domain (time-courses), the spatial domain (spatial maps), and the subject domain (subject modes). Spatial maps reflect brain regions of synchronous activations and deactivations, and subject modes reveal the strength of both these activations and deactivations; higher subjectmode values indicate higher activations and higher deactivations of the positive and negative parts of an IC, respectively. The number of ICs dimensions was estimated by MELODIC using the Laplace approximation to the Bayesian evidence of the model order (32). Spatial maps were thresholded modelling the probability of the noise class to $50 \%$ (using Gamma densities).

FMRI data set was decomposed into 18 independent components (IC). Afterwards, a selection of biologically relevant components was made, excluding the components that, according to the subject-mode analysis, appeared to be driven by outliers, as well as artifactual components produced by motion, high-frequency noise or vascular pulsations (6). Five ICs were finally selected, two of them associated with the task>control and three to the control>task contrast. Subsequently, we identified the main task-related components by computing spatial correlations between the average activation maps obtained with FEAT and the selected ICs. Z (Gaussianized T/F) statistic images were thresholded using clusters determined by $Z \geq 3$. IC1 was the task-related component that showed the greatest spatial correlation with the task 
pattern identified in the model-based analysis $(r=0.56)$. Specific information about the other selected components is included in the supplementary materials (supplementary materials 2).

\subsubsection{Cross-correlation connectivity analysis}

In order to investigate the connectivity between the main activated regions of the recognition memory component, we created eight spherical $8 \mathrm{~mm}$ regions of interest (ROI) centered on the peak voxels of the most relevant clusters from the main task component obtained from the model-free analysis (recognition memory network IC1).

From preprocessed fMRI data sets, the mean time-course was extracted from each of these ROls. Pearson's correlation coefficients were then computed between the time-courses from each pair of ROls and normalized using Fisher's $r$-to-Z transformation for each subject. A repeated-measures general linear mixed model (GLM) was used to test whether variables changed in each group across time. PASW-18 software was used to perform the statistical analyses.

The $\mathrm{fMRI}$ analysis pipeline is summarized in Figure 1.

-Insert Figure 1-

\section{RESULTS}

\subsection{Clinical variables and neuropsychological performance}

In the patient group, there were no statistically significant differences between baseline and follow-up levodopa equivalent daily doses (LEDD) and Hoehn and Yahr and UPDRS motor section scores. Moreover, GLM analysis showed a significant time effect on MMSE and group effect on BDI. No significant group-by-time interaction was found for these variables. NPI-Q did not show significant between-group differences or over-time changes (Table 2). 
Table 2. Clinical data and memory performance at baseline and at follow-up and time effect, group effect and group-by-time interaction.

\begin{tabular}{|c|c|c|c|c|c|c|c|}
\hline & \multicolumn{2}{|c|}{$\begin{array}{l}\text { PD (n=17) } \\
\text { Mean (SD) }\end{array}$} & \multicolumn{2}{|c|}{$\begin{array}{l}\text { Controls (n=13) } \\
\text { Mean (SD) }\end{array}$} & \multirow[t]{2}{*}{$\begin{array}{l}F \\
\text { (GxT) }\end{array}$} & \multirow[t]{2}{*}{$F(G)$} & \multirow[t]{2}{*}{$\begin{array}{l}F \\
(T)\end{array}$} \\
\hline & Baseline & Follow-up & Baseline & Follow-up & & & \\
\hline MMSE & $\begin{array}{l}29.53 \\
(0.51) \\
\end{array}$ & $\begin{array}{l}27.88 \\
(2.55) \\
\end{array}$ & $\begin{array}{l}29.92 \\
(0.28) \\
\end{array}$ & $\begin{array}{l}28.85 \\
(0.69) \\
\end{array}$ & 0.72 & 2.79 & $16.49 *$ \\
\hline BDI & \begin{tabular}{|l|}
7.53 \\
$(5.31)$
\end{tabular} & $\begin{array}{l}5.65 \\
(4.81)\end{array}$ & $\begin{array}{l}3.31 \\
(2.39)\end{array}$ & $\begin{array}{l}2.62 \\
(2.63)\end{array}$ & 0.59 & $7.50^{*}$ & 2.75 \\
\hline NPI & \begin{tabular}{|l|}
3.12 \\
$(5.38)$
\end{tabular} & $\begin{array}{l}2.00 \\
(1.90)\end{array}$ & $\begin{array}{l}1.08 \\
(1.44)\end{array}$ & $\begin{array}{l}1.46 \\
(1.66)\end{array}$ & 1.36 & 1.68 & 0.32 \\
\hline UPDRS & $\begin{array}{l}15.24 \\
(3.68)\end{array}$ & $\begin{array}{l}15.06 \\
(4.41)\end{array}$ & - & - & - & - & 0.25 \\
\hline Hoehn \& Yahr & \begin{tabular}{|l|}
1.77 \\
$(0.36)$ \\
\end{tabular} & $\begin{array}{l}1.85 \\
(0.42)\end{array}$ & - & - & - & - & 0.68 \\
\hline LEDD & \begin{tabular}{|l|}
277.65 \\
$(308.51)$ \\
\end{tabular} & $\begin{array}{l}430.59 \\
(350.78)\end{array}$ & - & - & - & - & 3.10 \\
\hline $\begin{array}{l}\text { RAVLT } \\
\text { learning }\end{array}$ & \begin{tabular}{|l|}
44.29 \\
$(12.20)$ \\
\end{tabular} & $\begin{array}{l}43.12 \\
(10.69)\end{array}$ & $\begin{array}{l}50.62 \\
(9.09)\end{array}$ & $\begin{array}{l}48.92 \\
(10.10)\end{array}$ & 0.03 & 2.79 & 0.85 \\
\hline $\begin{array}{l}\text { RAVLT } \\
\text { Delayed Recall }\end{array}$ & \begin{tabular}{|l|}
8.59 \\
$(3.50)$
\end{tabular} & $\begin{array}{l}9.41 \\
(3.30)\end{array}$ & $\begin{array}{l}10.39 \\
(2.29)\end{array}$ & $\begin{array}{l}9.92 \\
(2.75)\end{array}$ & 2.03 & 1.25 & 0.16 \\
\hline $\begin{array}{l}\text { RAVLT } \\
\text { Recognition }\end{array}$ & $\begin{array}{l}27.59 \\
(2.24)\end{array}$ & $\begin{array}{l}28.29 \\
(2.34)\end{array}$ & $\begin{array}{l}28.39 \\
(1.9)\end{array}$ & $\begin{array}{l}28.46 \\
(1.94)\end{array}$ & 0.88 & 0.45 & 1.37 \\
\hline
\end{tabular}

SD: standard deviation. The F values refer to those obtained with the repeated-measures general linear model. $F(G \times T)$ : group-by-time interaction; $F(G)$ : group effect; $F(T)$ : time effect. MMSE: Mini-mental state examination; BDI: Beck Depression Inventory-II; NPI: Neuropsychiatric Inventory; UPDRS: Unified Parkinson's disease rating scale, motor section; LEDD: Levodopa equivalent daily dose; RAVLT: Rey's Auditory Verbal Learning Test. *Significant at $p$ value $\leq 0.05$

Verbal memory performance, assessed by RAVLT, did not show any statistically significant effects for any of the variables analyzed (Table 2).

Supplementary materials 3 show the results of the other neuropsychological tests used. Significant group differences were observed for semantic fluency and backward digit span. A 
significant group-by-time interaction was observed for forward digit span, resulting from a slight worsening in controls' and a slight improvement in PD patients' performances over time.

\subsection{Performance in the $\mathrm{fMRI}$ recognition memory task}

In the $\mathrm{fMRI}$ recognition memory task performance, we found several significant time effects. Both patients and controls performed worse in the correct-reject responses at follow-up, but the worsening was similar in both groups (group effect and group-by-time interaction were not significant). The main differences between groups were observed for false-positive responses. For this variable, a significant group effect was seen - patients' performance was worse than controls', and both groups presented a significant over-time decline. In addition, the group-bytime interaction showed a trend to significance, indicating that the decline in this ability was more marked in patients.

Table 3. fMRI performance at baseline and at follow-up, time effect, group effect and groupby-time interaction.

\begin{tabular}{|c|c|c|c|c|c|c|c|}
\hline & \multicolumn{2}{|c|}{$\begin{array}{l}\text { PD }(\mathbf{n}=\mathbf{1 7}) \\
\text { mean (SD) }\end{array}$} & \multicolumn{2}{|c|}{$\begin{array}{l}\text { Controls }(\mathbf{n}=\mathbf{1 3}) \\
\text { mean }(S D)\end{array}$} & \multirow[t]{2}{*}{$\begin{array}{l}F \\
(G \times T)\end{array}$} & \multirow[t]{2}{*}{$\begin{array}{l}F \\
(G) \\
\end{array}$} & \multirow[t]{2}{*}{$\begin{array}{l}F \\
(T)\end{array}$} \\
\hline & Baseline & Follow-up & Baseline & Follow-up & & & \\
\hline Hits & $\begin{array}{l}54.69 \\
(5.70)\end{array}$ & $\begin{array}{l}54.94 \\
(7.17)\end{array}$ & $\begin{array}{l}54.85 \\
(5.69)\end{array}$ & $\begin{array}{l}56.62 \\
(6.60)\end{array}$ & 0.16 & 0.29 & 0.24 \\
\hline $\begin{array}{l}\text { Correct } \\
\text { Rejects }\end{array}$ & $\begin{array}{l}59.57 \\
(9.98)\end{array}$ & $\begin{array}{l}49.71 \\
(20.04)\end{array}$ & $\begin{array}{l}63.23 \\
(9.09)\end{array}$ & $\begin{array}{l}60.15 \\
(6.69)\end{array}$ & 1.56 & 3.01 & $5.68^{*}$ \\
\hline $\begin{array}{l}\text { False } \\
\text { positives }\end{array}$ & $\begin{array}{l}11.31 \\
(10.20)\end{array}$ & $\begin{array}{l}27.19 \\
(26.53)\end{array}$ & $\begin{array}{l}6.77 \\
(9.09) \\
\end{array}$ & $\begin{array}{l}9.85 \\
(6.69)\end{array}$ & $3.44^{+}$ & $7.55^{*}$ & $5.13^{*}$ \\
\hline Missings & $\begin{array}{l}15.31 \\
(7.17)\end{array}$ & $\begin{array}{l}16.06 \\
(8.37)\end{array}$ & $\begin{array}{l}15.15 \\
(5.70)\end{array}$ & $\begin{array}{l}14.39 \\
(6.50)\end{array}$ & 0.16 & 0.24 & 0.01 \\
\hline $\begin{array}{l}\text { Reaction } \\
\text { time }\end{array}$ & $\begin{array}{l}687.54 \\
(152.83)\end{array}$ & $\begin{array}{l}636.37 \\
(134.28)\end{array}$ & $\begin{array}{l}640.05 \\
(83.75)\end{array}$ & $\begin{array}{l}644.01 \\
(70.12)\end{array}$ & 1.70 & 0.24 & 2.32 \\
\hline
\end{tabular}

SD: standard deviation. The F values refer to those obtained with the repeated-measures general linear model. $F(G \times T)$ : group-by-time interaction; $F(G)$ : group effect; $F(T)$ : time effect. ${ }^{*} p \leq 0.05,+p=0.07$. 


\section{3 fMRI data results}

\subsubsection{Model-based analyses}

In figure 2 are depicted the areas involved in the recognition memory task for both groups. In both patients and controls the regions that achieved significant activations during the task were located mainly in cortical areas including the occipital lobes, bilateral orbitofrontal cortices and anterior paracingulate region (see also supplementary material 4), but also included subcortical structures such as the left thalamus and the basal ganglia. This pattern was used to identify the main task-related component in the model-free analysis by calculating spatial correlations. Intergroup comparisons revealed no significant results.

-Insert figure 2-

\subsubsection{Model-free analysis}

From the five task-related components identified (see Supplementary materials 1), IC1 was the only to show a decreased activity over time in PD patients $(p<0.04)$. This independent component had a left-hemisphere predominance and was characterized by activations in bilateral orbitofrontal regions, bilateral middle frontal gyri, bilateral frontal poles, anterior paracingulate regions, bilateral superior parietal lobes and the posterior region of the left middle temporal gyrus. In the left frontal lobe, a single cluster of activation, with a peak in the left orbitofrontal area, included all of the above-mentioned frontal regions. IC1 also involved deactivations in DMN areas including the anterior part of the paracingulate gyrus and the precuneus (Figure 3 and also supplementary material 5).

-Insert figure 3- 
A correlation analysis was performed between IC1 and individual activations-deactivations (subject modes) and recognition task results. There was a significant correlation between PD patients' false-positive errors and IC1 subject modes $(r=-0.403, p=0.027)$, as well as with its complementary measure, the correct rejects $(r=0.403, p=0.027)$. In the control group we did not observe any significant correlations.

\subsection{Cross-correlation connectivity analysis}

Main spherical ROls of $8 \mathrm{~mm}$ radius included right middle frontal gyrus (RMFG), right superior parietal lobe (RSPL), right frontal pole (RFP), right orbitofrontal cortex (ROFC), anterior paracingulate cortex (APC), left superior parietal lobe (LSPL), left orbitofrontal cortex (LOFC), left middle temporal gyrus (LMTG). Longitudinal analyses of correlation coefficients between ROls showed some significant effects indicating deterioration of functional connectivity between specific regions of IC1 in PD patients. In this group, there was a decrease in functional connectivity (weaker positive correlation) between RMFG and bilateral SPL, whereas the opposite effect was found in controls. Moreover, whereas controls' connectivity between RMFG and RFP decreased at follow-up, PD patients' remained stable (Table 5 and Figure 4).

Table 5. Correlation between verbal recognition network ROls, at baseline and at follow-up, time effect, group effect and group-by-time interaction.

\begin{tabular}{|l|l|l|l|l|l|l|l|}
\hline & \multicolumn{2}{|l|}{$\begin{array}{l}\text { PD (n=17) } \\
\text { mean (SD) }\end{array}$} & \multicolumn{2}{l|}{$\begin{array}{l}\text { Controls (n=13) } \\
\text { mean (SD) }\end{array}$} & $\begin{array}{l}\text { F } \\
\text { (GxT) }\end{array}$ & $\begin{array}{l}\text { F } \\
\text { (G) }\end{array}$ & $\begin{array}{l}\text { F } \\
\text { (T) }\end{array}$ \\
\hline & Baseline & Follow-up & Baseline & Follow-up & & & \\
\hline RMFG-RSPL & $\begin{array}{l}0.50 \\
(0.26)\end{array}$ & $\begin{array}{l}0.33 \\
(0.20)\end{array}$ & $\begin{array}{l}0.30 \\
(0.22)\end{array}$ & $\begin{array}{l}0.42 \\
(0.29)\end{array}$ & $8.38^{*}$ & 0.52 & 0.31 \\
\hline RMFG-RFP & $\begin{array}{l}0.26 \\
(0.33)\end{array}$ & $\begin{array}{l}0.29 \\
(0.25)\end{array}$ & $\begin{array}{l}0.47 \\
(0.14)\end{array}$ & $\begin{array}{l}0.29 \\
(0.17)\end{array}$ & $4.98^{*}$ & 1.92 & 2.38 \\
\hline RMFG-LSPL & $\begin{array}{l}0.45 \\
(0.24)\end{array}$ & $\begin{array}{l}0.25 \\
(0.16)\end{array}$ & $\begin{array}{l}0.31 \\
(0.18)\end{array}$ & $\begin{array}{l}0.42 \\
(0.30)\end{array}$ & $8.09^{*}$ & 0.62 & 0.62 \\
\hline RFP-LOFC & $\begin{array}{l}0.24 \\
(0.20)\end{array}$ & $\begin{array}{l}0.10 \\
(0.22)\end{array}$ & $\begin{array}{l}0.32 \\
(0.15)\end{array}$ & $\begin{array}{l}0.25 \\
(0.14)\end{array}$ & 1.13 & $4.34^{*}$ & $6.77^{*}$ \\
\hline ROFC-LOFC & $\begin{array}{l}0.37 \\
(0.17)\end{array}$ & $\begin{array}{l}0.24 \\
(0.25)\end{array}$ & $\begin{array}{l}0.51 \\
(0.22)\end{array}$ & $\begin{array}{l}0.37 \\
(0.16)\end{array}$ & 0.04 & 3.87 & $13.67^{*}$ \\
\hline
\end{tabular}


Mean values are Pearson's correlation coefficients normalized using Fisher's r-to-Z transformation. SD: standard deviation. The $\mathrm{F}$ values refer to those obtained with a repeated-measures general linear model. $F(G \times T)$ : group-by-time interaction; $F(G)$ : group effect; $F(T)$ : time effect; RMFG: right middle frontal gyrus; RSPL: right superior parietal lobe; RFP: right frontal pole; ROFC: right orbitofrontal cortex; APC: anterior paracingulate cortex; LSPL: left superior parietal lobe; LOFC: left orbitofrontal cortex; LMTG: left middle temporal gyrus. ${ }^{*} p \leq 0.05$

Correlation strength between RFP and LOFC was significantly lower in PD patients than in controls. A significant over-time decline in correlation strength between these structures was observed in both groups, with no significant group-by-time interaction. The strength of correlation between right and left OFC also decreased over time in both groups, with no significant group effect or group-by-time interaction (Table 5). No other significant effects in the analyses of correlations between the selected ROIs were detected.

Finally, correlation strength between right and left OFC decreased over time in both groups (Table 5). No other significant effects between the selected ROIs were detected.

-Insert figure 4-

\section{DISCUSSION}

In this study, we observed that PD patients presented a progressive loss in the recognition memory network's pattern of activation and deactivation; moreover, the results revealed deterioration in the strength of connectivity between the main areas involved in this network.

In this study with an average follow-up of 35.5 months, PD patients remained stable in learning and memory as assessed by clinical neuropsychological tests. However, we observed a decline in the $\mathrm{fMRI}$ recognition task performance. The increase in false-positive responses at follow-up was significantly more marked in the PD patients group than in controls. This finding is in agreement with Whittington et al., (4) who demonstrated that the recognition impairment in 
non-demented PD is seen in tasks of high demand (increased number of items). While in the RAVLT recognition part the number of stimuli is only 30 , in the fMRI task there were 70 stimuli; this may have allowed the detection of subtle neuropsychological deficits not identified through standard clinical testing.

The regions that we have found to be activated during the recognition memory task, involving both patients and controls, included several cortical areas (occipital lobes, bilateral orbitofrontal cortices and anterior paracingulate) and also subcortical structures involving bilaterally the basal ganglia and the left thalamus. However, the longitudinal data analyses showed that PD patients had a decreased task-related activity over time in cortical regions (IC1), but not in subcortical ones. Specifically, middle frontal gyrus, frontal pole, orbitofrontal cortex, anterior paracingulate gyrus, superior parietal lobe and the posterior portion of the middle temporal gyrus. All of these areas have been previously related to the recognition memory network $(8,33)$. Indeed, the cortical activation pattern observed in the current study is similar to those reported by Spaniol et al. (34) after an fMRI meta-analysis. In all the studies included in this meta-analysis, the activated regions were prefrontal and parietal bilaterally, with left-hemispheric predominance.

Regarding the pattern of deactivation detected in the recognition memory network, we found a decreased task-related deactivation over time in PD patients, specifically in the anterior part of the paracingulate gyrus and the precuneus. Both these areas are part of the $\operatorname{DMN}(35,36)$. In our previous study, we detected that PD patients, compared to controls, failed to deactivate the precuneus during the recognition memory task (8). Impaired deactivation of the precuneus in PD was also reported during the performance of a card-sorting task (15). There are no previous longitudinal studies of $\mathrm{DMN}$ dysfunctions in PD, but abnormal patterns of deactivation in DMN have been previously reported as an indicator of progression in other neurodegenerative diseases (37). 
PD patients in our study committed more false-positive errors in the fMRI task, and with significantly more marked over-time worsening, than controls. These false-positive errors correlated with the pattern of activation and deactivation in the recognition memory network, suggesting that an appropriate recruitment of the recognition memory network entails a better performance. In line with these results, Van Eimeren et al. (15) found a positive correlation between errors in an executive task and BOLD signal change in specific regions of the DMN such as precuneus and posterior cingulate cortex, meaning that, the more deactivated those areas were, the better the performance.

The cross-correlation analysis approach allowed us to evaluate the variations in connectivity within the recognition memory network between baseline and follow-up. Previous PD crosssectional studies showed abnormal connectivity patterns during cognitive tasks $(14,15)$. Van Eimeren et al. (15) studied the cortical deactivation during an executive functions task as well as the functional connectivity among main areas of interest. Their results suggest functional fronto-striatal disconnection in PD patients.

In the present study, the connectivity analysis between main regions of interest revealed that PD patients showed a progressive decrease in frontoparietal connectivity whereas the connectivity between frontal areas remained stable. Functional disconnection in PD may result from the primary synucleinopathy and the synaptic dysfunction associated with it (38) or may be due to white matter microstructural damage, described in PD patients using diffusion tensor imaging techniques (39-41). The decreased fronto-parietal connectivity could be mediated by the degeneration of tracts that connect these regions, such as the superior longitudinal fasciculus or the inferior fronto-occipital fasciculus, which have been described to be affected in non-demented PD patients $(40,42)$.

The fact that, in PD patients, the connectivity between right frontal regions remained stable, whereas in controls it declined, could be linked to the loss of connectivity between frontal and 
parietal regions observed in the former. We can speculate that the affectation of long-range connections could result in an increase in more local circuits as a functional compensation mechanism. Further studies would be necessary, however, to evaluate this hypothesis.

Fronto-parietal connectivity may play an important role in PD-related cognitive deficits, as previous work has found a cognitively relevant network in PD involving these areas. In a crosssectional study, Huang et al. (19) identified a cognition-related metabolic pattern in the network analysis of FDG PET scans from 15 non-demented PD patients with mild to moderate motor symptoms. This pattern was characterized by relative hypometabolism of the dorsolateral prefrontal cortex, rostral supplementary motor area (preSMA) and superior parietal regions, associated with relative cerebellar/dentate nucleus metabolic increases. This network expression correlated significantly with indices of memory and executive functioning. The same authors performed a longitudinal FDG PET study of PD patients without evident cognitive impairment. In accordance with our results, they found that disease progression was associated with declining metabolism in the prefrontal and parietal regions. Similarly, Carbon et al. (43) found longitudinal changes in sequence learning performance and associated taskrelated cerebral blood flow (H2 150 PET) in non-demented PD patients. After a two-year follow-up, significant declines in learning-related activation were detected in parietal and temporo-occipital association areas and in the right dorsolateral prefrontal cortex.

In spite of previous evidence, the progression of functional brain changes in PD patients remains controversial. In fact, after a longer follow-up period (4 years), Huang et al. (18) found an inverse result, that is, an increase in cognition-related metabolic pattern similar to motorrelated metabolic pattern progression. The authors related this result to incipient cognitive impairment. Contrarily, Bohnen et al. (44) found a decrease in metabolic activity (FDG PET) in a small sample of patients who converted to PD with dementia (PDD) after two years of followup. The metabolic reduction pattern included the thalamus and posterior cingulate, occipital, 
parietal and frontal areas, with mild reduction in the temporal lobe. The most prominent metabolic reduction in PDD was seen in the cuneus and precuneus and in mesiofrontal areas. In the future, progression of connectivity patterns between frontoparietal regions should be studied after longer follow-up periods.

The subjects in our study did not present clinically significant cognitive impairments; however PD patients frequently present cognitive deficits. Specifically, visuospatial/visuoperceptual deficits, which, in severe cases, could interfere with the performance of tasks such as the recognition memory paradigm used in this study, for that reason this issue should ideally be controlled in future studies. We also have to point out that all PD patients included in the sample were assessed under their usual antiparkinsonian medication. In order to consider potentially confounding effects of dopaminergic medications, in future studies we should address the same question on drug abstinent or naïve patients to elucidate the influence of the dopaminergic medication on the activation of cerebral areas related to recognition memory. Although the PD patients in our sample presented normal average BDI scores, these were higher than controls'; future studies should try to pair groups by severity of depressive symptoms, taking into account the effect of antidepressant medication. Finally, we have also to consider the drop-out of subjects from the initial sample to follow up, a common limitation in longitudinal studies. However, the comparisons between the initial sample and the one included in the longitudinal assessment, either for controls or for PD patients, showed no significant differences in demographical and clinical characteristics. Even so, in the future our study should be replicated with a bigger sample.

In conclusion, our results show a decrease in the recognition memory network activation pattern over time and an abnormal connectivity pattern between main regions involved in this memory network in PD patients. To the best our knowledge, this is the first longitudinal study to reveal a progressive decrease in functional connectivity between areas involved in a cognitive network in PD patients. 


\section{Acknowledgements:}

Without the support of the patients, their families, and control subjects this work would have not been possible. We also acknowledge the invaluable help of Silvia Juanes (University of Barcelona) for the support on the statistical analyses.

\section{Funding statement:}

This study was supported by the PSI2010-16174 from the Spanish government to C.J., H.C.B. and B.S., and by Generalitat de Catalunya [2009 SGR0836 to E.T., 2009 SGR0941 to C.J. and an FI-DGR grant (2011FI_B 00045) to H.C.B., and CIBERNED.

\section{Disclosure Statement:}

All authors have contributed to the project and production of the manuscript and there is no conflict of interest.

\section{Author's contribution:}

1A conception and design, 1B acquisition of data, 1C analysis and interpretation of data;

2A drafting the article 2B revising it critically for important intellectual content

3 final approval of the version to be published.

Bàrbara Segura : 1C, 2A, 2B, 3

Naroa Ibarretxe-Bilbao: 1B, 2B, 3

Roser Sala-Llonch: 1C, 2A, 2B, 3

Hugo Cesar Baggio: 1C, 2A, 2B, 3

Maria Josep Martí: 1B, 1C, 2B, 3 
Francesc Valldeoriola: 1B, 1C, 2B, 3

Pere Vendrell: 1C, 2B, 3

Nuria Bargalló: 1C, 2B, 3

Eduard Tolosa: 1A, 1C, 2A, 2B, 3

Carme Junqué: 1A, 1C, 2A, 2B, 3 


\section{REFERENCES}

1. Aarsland D, Bronnick K, Larsen JP, et al. Cognitive impairment in incident, untreated Parkinson disease: the Norwegian ParkWest study. Neurology 2009;72:1121-6.

2. Elgh $E$, Domellof $M$, Linder J, et al. Cognitive function in early Parkinson's disease: a population-based study. Eur J Neurol 2009;16:1278-84

3. Higginson $\mathrm{Cl}$, Wheelock VL, Carroll KE et al. Recognition memory in Parkinson's disease with and without dementia: evidence inconsistent with the retrieval deficit hypothesis. J Clin Exp Neuropsychol 2005;27:516-28.

4. Whittington CJ, Podd J, Stewart-Williams S. Memory deficits in Parkinson's disease. J Clin Exp Neuropsychol 2006;28:738-54.

5. Li K, Guo L, Nie J et al. Review of methods for functional brain connectivity detection using fMRI. Comput Med Imaging Graph 2009;33:131-9.

6. Beckmann CF, Smith SM. Tensorial extensions of independent component analysis for multisubject FMRI analysis. Neuroimage 2005;25:294-311.

7. Bai F, Shi Y, Yuan Y et al. Altered self-referential network in resting-state amnestic type mild cognitive impairment. Cortex 2012;48:604-13.

8. Ibarretxe-Bilbao N, Zarei M, Junque C, Marti MJ, Segura B, Vendrell P, et al. Dysfunctions of cerebral networks precede recognition memory deficits in early Parkinson's disease. Neuroimage 2011;57:589-97.

9. Buckner RL, Andrews-Hanna JR, Schacter DL. The brain's default network: anatomy, function, and relevance to disease. Ann N Y Acad Sci 2008;1124:1-38.

10. Jahanshahi $\mathrm{M}$, Jones $\mathrm{CR}$, Zijlmans J, et al. Dopaminergic modulation of striato-frontal connectivity during motor timing in Parkinson's disease. Brain 2010;133:727-45.

11. Palmer SJ, Li J, Wang ZJ, et al. Joint amplitude and connectivity compensatory mechanisms in Parkinson's disease. Neuroscience 2010;166:1110-18.

12. Wu T, Chan P, Hallett M. Effective connectivity of neural networks in automatic movements in Parkinson's disease. Neuroimage 2010;49:2581-87.

13. Wu T, Wang L, Hallett $M$, et al. Effective connectivity of brain networks during selfinitiated movement in Parkinson's disease. Neuroimage 2011;55:204-15.

14. Rowe J, Stephan KE, Friston K, et al. Attention to action in Parkinson's disease: impaired effective connectivity among frontal cortical regions. Brain 2002;125:276-89.

15. van Eimeren T, Monchi O, Ballanger B, et al. Dysfunction of the default mode network in Parkinson disease: a functional magnetic resonance imaging study. Arch Neurol 2009;66:877-83. 
16. Wu T, Wang L, Chen Y, et al. Changes of functional connectivity of the motor network in the resting state in Parkinson's disease. Neurosci Lett 2009;460:6-10.

17. Helmich RC, Derikx LC, Bakker M, et al. Spatial remapping of cortico-striatal connectivity in Parkinson's disease. Cereb Cortex 2010;20:1175-86.

18. Huang C, Tang C, Feigin A, et al. Changes in network activity with the progression of Parkinson's disease. Brain 2007;130:1834-46.

19. Huang $C$, Mattis $P$, Tang $C$, et al. Metabolic brain networks associated with cognitive function in Parkinson's disease. Neuroimage 2007;34:714-23.

20. Ibarretxe-Bilbao N, Junque C, Marti MJ, et al. Olfactory impairment in Parkinson's disease and white matter abnormalities in central olfactory areas: A voxel-based diffusion tensor imaging study. Mov Disord 2010;25:1888-94.

21. Tomlinson $\mathrm{CL}$, Stowe $\mathrm{R}$, Patel $\mathrm{S}$, et al. Systematic review of levodopa equivalency reporting in Parkinson's disease. Mov Disord 2010;25:2649-53.

22. Daniel SE, Lees AJ. Parkinson's Disease Society Brain Bank, London: overview and research. J Neural Transm Suppl 1993;39:165-72.

23. Dubois B, Burn D, Goetz C, et al. Diagnostic procedures for Parkinson's disease dementia: recommendations from the movement disorder society task force. Mov Disord 2007;22:2314-24.

24. Beck AT, Steer RA, Brown GK. Manual for the Beck Depression Inventory-II. San Antonio, TX: Psychological Corporation 1996.

25. Cummings JL, Mega M, Gray K, et al. The Neuropsychiatric Inventory: comprehensive assessment of psychopathology in dementia. Neurology 1994;44:2308-14.

26. Rey A. El Examen Clínico en Psicología. Editorial Kapelusz. Buenos Aires, Argentina: Editorial Kapelusz 1962.

27. Jenkinson $M$, Bannister $P$, Brady $M$, et al. Improved optimization for the robust and accurate linear registration and motion correction of brain images. Neuroimage 2002;17:825-41.

28. Smith SM. Fast robust automated brain extraction. Hum Brain Mapp 2002;17:143-55.

29. Jenkinson M, Smith S. A global optimisation method for robust affine registration of brain images. Med Image Anal 2001;5:143-56.

30. Beckmann CF, Jenkinson M, Smith SM. General multilevel linear modeling for group analysis in FMRI. Neuroimage 2003;20:1052-63.

31. Woolrich MW, Behrens TE, Beckmann CF, et al. Multilevel linear modelling for FMRI group analysis using Bayesian inference. Neuroimage 2004;21:1732-47. 
32. Beckmann CF, Smith SM. Probabilistic independent component analysis for functional magnetic resonance imaging. IEEE Trans Med Imaging 2004;23:137-52.

33. Cabeza R, Nyberg L. Imaging cognition II: An empirical review of 275 PET and fMRI studies. J Cogn Neurosci 2000;12:1-47.

34. Spaniol J, Davidson PS, Kim AS, et al. Event-related fMRI studies of episodic encoding and retrieval: meta-analyses using activation likelihood estimation. Neuropsychologia 2009;47:1765-79.

35. Greicius MD, Krasnow B, Reiss AL, et al. Functional connectivity in the resting brain: a network analysis of the default mode hypothesis. Proc Natl Acad Sci U S A 2003;100:253-8.

36. Raichle ME, MacLeod AM, Snyder AZ, et al. A default mode of brain function. Proc Natl Acad Sci U S A 2001;98:676-82.

37. Petrella JR, Sheldon FC, Prince SE, et al. Default mode network connectivity in stable vs progressive mild cognitive impairment. Neurology 2011;76:511-7.

38. Schulz-Schaeffer WJ. The synaptic pathology of alpha-synuclein aggregation in dementia with Lewy bodies, Parkinson's disease and Parkinson's disease dementia. Acta Neuropathol 2010;120:131-43.

39. Karagulle-Kendi AT, Lehericy $S$, Luciana $M$, et al. Altered diffusion in the frontal lobe in Parkinson disease. AJNR Am J Neuroradiol 2008;29:501-5.

40. Gattellaro G, Minati L, Grisoli M, et al. White matter involvement in idiopathic Parkinson disease: a diffusion tensor imaging study. AJNR Am J Neuroradiol 2009;30: 1222-6.

41. Rae $\mathrm{CL}$, Correia MM, Altena E, et al. White matter pathology in Parkinson's disease: The effect of imaging protocol differences and relevance to executive function. Neuroimage 2012;62:1675-84.

42. Baggio HC, Segura B, Ibarretxe-Bilbao N, et al. Structural correlates of facial emotion recognition deficits in Parkinson's disease patients. Neuropsychologia 2012;50:2121-8.

43. Carbon M, Reetz K, Ghilardi MF, et al. Early Parkinson's disease: longitudinal changes in brain activity during sequence learning. Neurobiol Dis 2010;37:455-60.

44. Bohnen NI, Koeppe RA, Minoshima S, et al. Cerebral glucose metabolic features of Parkinson disease and incident dementia: longitudinal study. J Nucl Med 2011;52:84855. 


\section{Figures legends:}

Figure 1. fMRI data preprocessing and analysis.

Figure 2. fMRI model-based results. Average activation map (recognition task>control condition) was obtained from all the subjects (PD patients and control group). Results are corrected at $\mathrm{p}<0.05$ for multiple comparisons.

Figure 3. A. Spatial pattern of IC 1 (recognition task > control) including activation areas (warm colors) and deactivation areas (cold colors) the PD patients showed a decrease activity in IC 1 group over time $(p<0.04)$. B. Regions of interest (ROI) (green) from peak voxels of task related activation areas in IC1, selected to perform cross-correlation study (seed-to-seed connectivity). C. Time-courses represented the temporal profile of IC1 across group (red line) overlaid on the task-paradigm (block design) (green line).

Figure 4. Results of cross-correlation functional connectivity analysis (seed-to-seed connectivity). Functional connectivity between fronto-parietal ROls was decreased; frontofrontal connectivity was maintained in PD. z: Fisher r-to-Z conversion. PD: Parkinson's disease patients. HC: Healthy controls. Bs: Base-line. Fw: Follow-up. RMFG: right middle frontal gyrus; RSPL: right superior parietal lobe; RFP: right frontal pole; ROFC: right orbitofrontal cortex; APC: anterior paracingulate cortex; LSPL: left superior parietal lobe; LOFC: left orbitofrontal cortex; LMTG: left middle temporal gyrus

\section{Appendices material legends:}

Supplementary material 1.

Sociodemographic characteristics of subjects at baseline for the initially recruited sample and the final sample included in the longitudinal assessment, according to group. Student's t-test 
statistics and significance value for sample comparisons according to group are shown. MMSE: Mini-mental state examination; BDI: Beck's depression inventory-II; NPI: Cumming's neuropsychiatric inventory; LEDD: levodopa daily equivalent dose; Disease duration: duration in years of motor symptoms; Age at onset: age of appearance of first motor symptoms.

Supplementary material 2

Relevant components selected from model-free analysis results. Control-condition related components included IC2, IC3 and IC5 (recognition task<control condition). Recognition memory task related component is IC4 (recognition task>control condition). These ICs did not show time or group effect. (Left) Spatial patterns of IC2, IC3, IC4 and IC5, including activation areas (warm colors) and deactivation areas (cold colors). (Center) Time-courses represented the temporal profile of ICs across group (red line) overlaid on the task-paradigm (block design) (green line). (Right) Peak voxels activation/deactivations regions involved in each component. L: Left, R: Right, B: Bilateral.

Supplementary material 3

Neuropsychological performance at baseline and at follow-up and time effect, group effect and group-by-time interaction. SD: standard deviation. The F values refer to those obtained with the repeated-measures general linear model. $F(G \times T)$ : group-by-time interaction; $F(G)$ : group effect; $F(T)$ : time effect.

Supplementary material 4. Local maxima of significant cluster list obtained from model based FEAT analysis. LOP: Left Occpital Pole LOFC: left orbitofrontal cortex; ROFC: right orbitofrontal cortex; APC: anterior paracingulate cortex.

Supplementary material 5. 
Local maxima of significant cluster list obtained from activation areas that showed decreased activity in IC1 for the PD group over time ( $<0.04)$. RMFG: right middle frontal gyrus; RSPL: right superior parietal lobe; RFP: right frontal pole; ROFC: right orbitofrontal cortex; APC: anterior paracingulate cortex; LSPL: left superior parietal lobe; LOFC: left orbitofrontal cortex; LMTG: left middle temporal gyrus. 


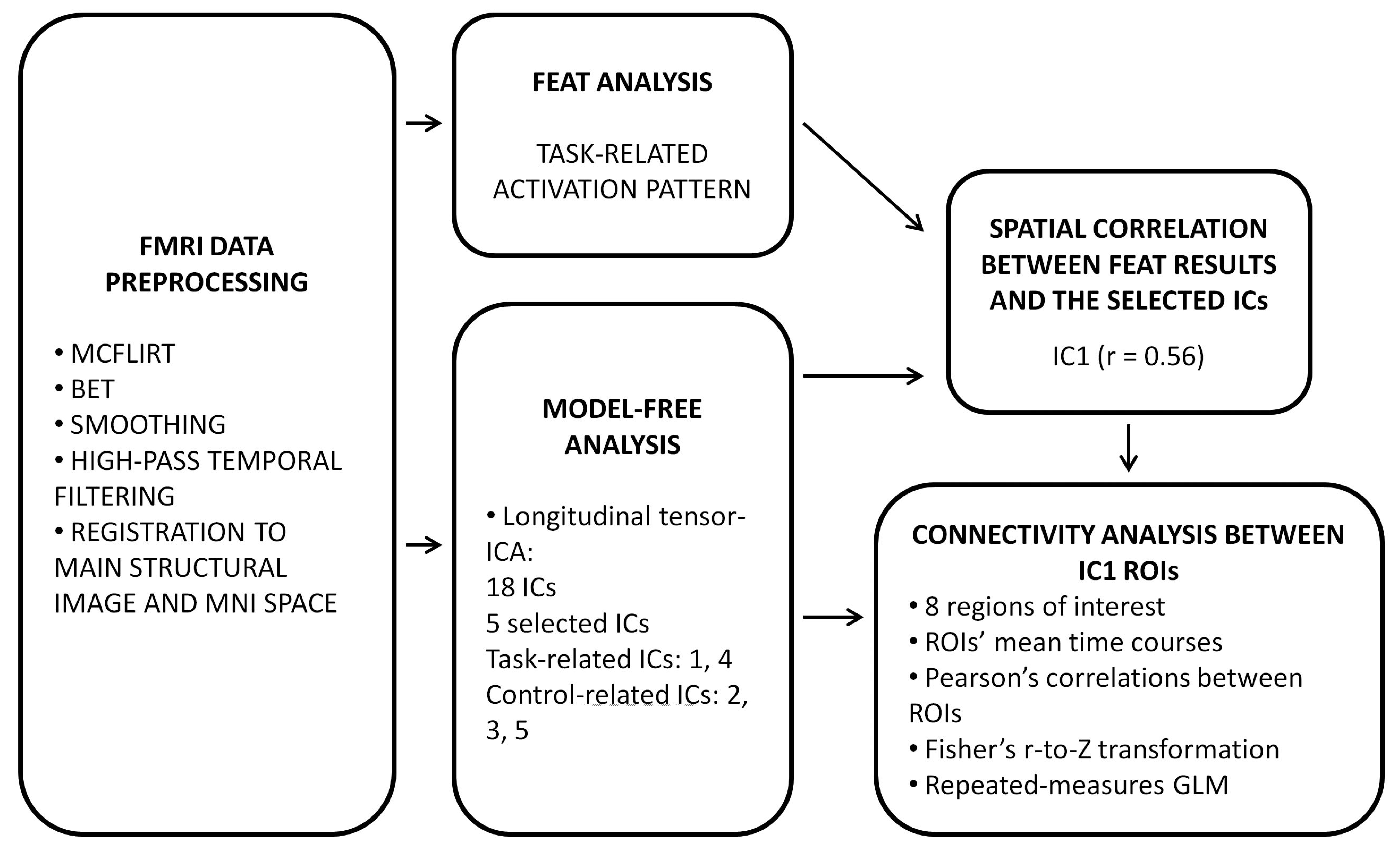



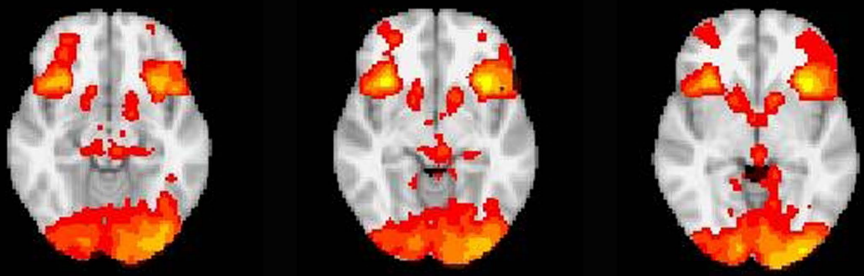

R

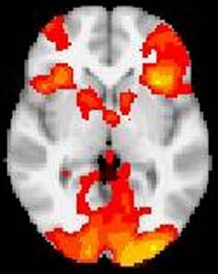

R

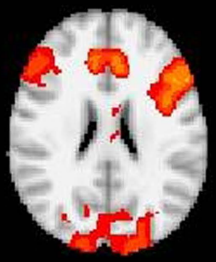

R
R

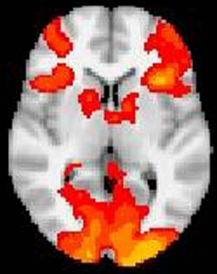

R

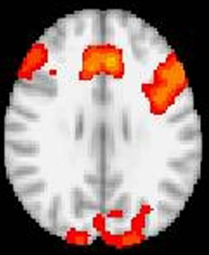

R
R

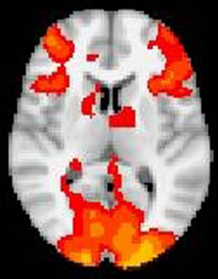

R

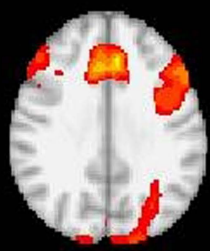

R 

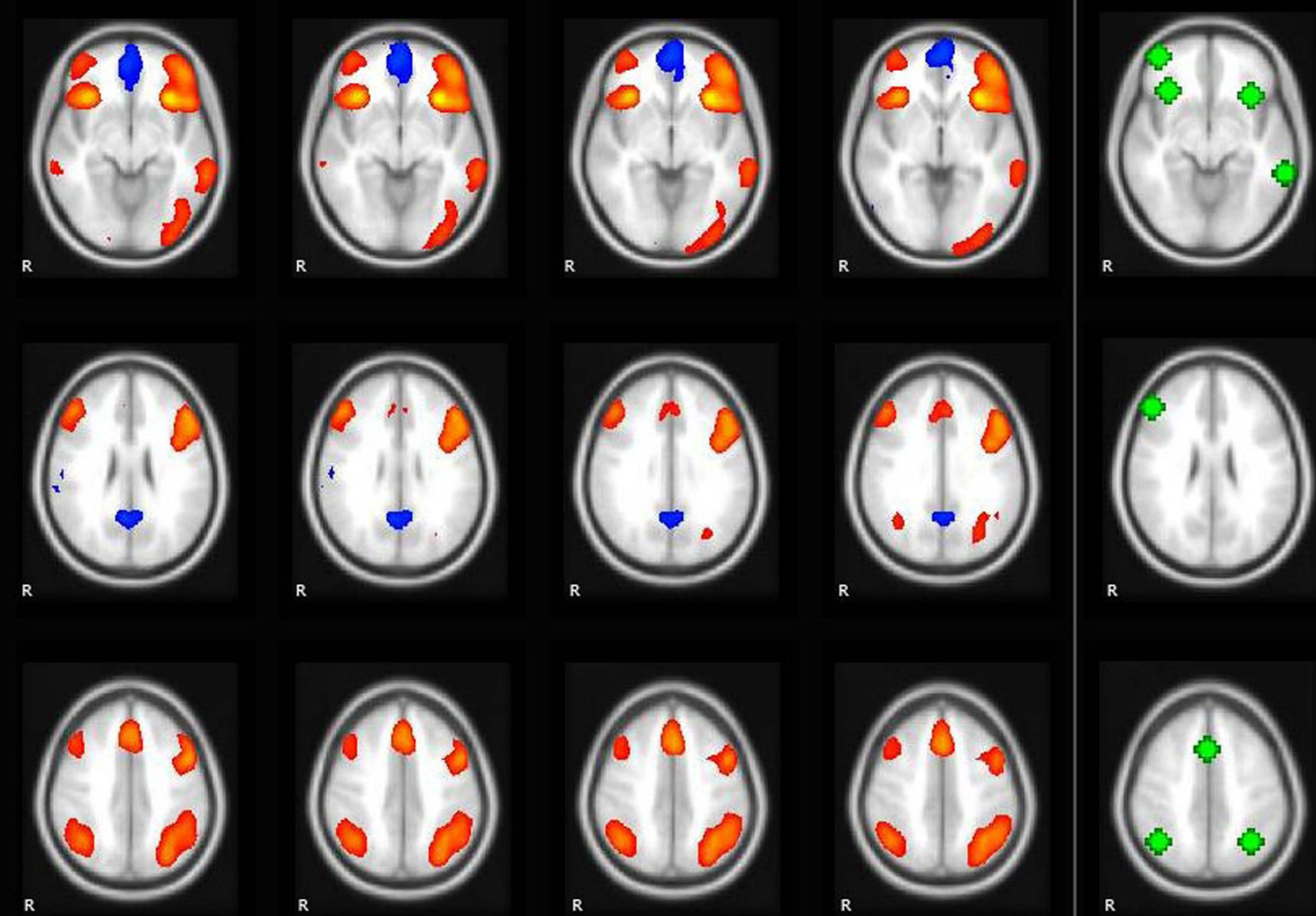

A 


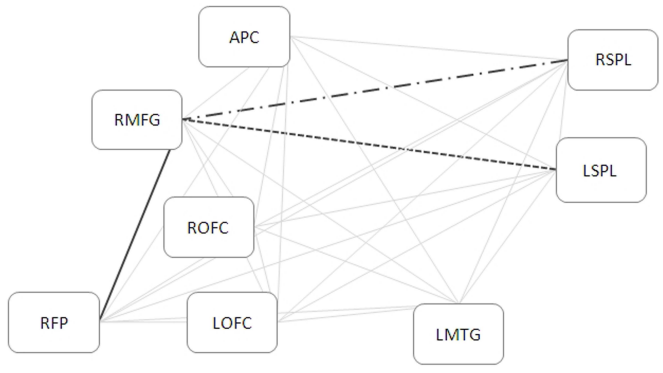

-.- mean $\mathrm{z}_{\mathrm{blPD}}=0,502(\mathrm{SD}=0,263)$; mean $\mathrm{z}_{\mathrm{fwPD}}=0,327(\mathrm{SD}=0,199)$ mean $\mathrm{z}_{\mathrm{bl} \mathrm{HC}}=0,302(\mathrm{SD}=0,221)$; mean $\mathrm{z}_{\mathrm{fw}} \mathrm{HC}=0,420(\mathrm{SD}=0,294)$ mean $\mathrm{z}_{\mathrm{blPD}}=0,450(\mathrm{SD}=0,242) ;$ mean $\mathrm{z}_{\mathrm{fwPD}}=0,246(\mathrm{SD}=0,158)$ mean $\mathrm{z}_{\mathrm{blHc}}=0,305(\mathrm{SD}=0,181)$; mean $\mathrm{z}_{\mathrm{fw} H \mathrm{HC}}=0,421(\mathrm{SD}=0,304)$ mean $\mathrm{Z}_{\mathrm{bl} \mathrm{PD}}=0,261(\mathrm{SD}=0,330)$; mean $\mathrm{z}_{\mathrm{fw}} \mathrm{PD}=0,289(\mathrm{SD}=0,249)$ mean $\mathrm{z}_{\mathrm{bl} \mathrm{HC}}=0,473(\mathrm{SD}=0,140)$; mean $\mathrm{z}_{\mathrm{w} \mathrm{HC}}=0,288(\mathrm{SD}=0,174)$ 


\section{Appendices material legends:}

Supplementary material 1.

Sociodemographic characteristics of subjects at baseline for the initially recruited sample and the final sample included in the longitudinal assessment, according to group. Student's t-test statistics and significance value for sample comparisons according to group are shown. MMSE: Mini-mental state examination; BDI: Beck's depression inventory-II; NPI: Cumming's neuropsychiatric inventory; LEDD: levodopa daily equivalent dose; Disease duration: duration in years of motor symptoms; Age at onset: age of appearance of first motor symptoms.

Supplementary material 2

Relevant components selected from model-free analysis results. Control-condition related components included IC2, IC3 and IC5 (recognition task<control condition). Recognition memory task related component is IC4 (recognition task>control condition). These ICs did not show time or group effect. (Left) Spatial patterns of IC2, IC3, IC4 and IC5, including activation areas (warm colours) and deactivation areas (cold colours). (Center) Time courses represented the temporal profile of ICs across group (red line) overlaid on the task-paradigm (block design) (green line). (Right) Peak voxels activation/deactivations regions involved in each component. L: Left, R: Right, B: Bilateral.

Supplementary material_3 Neuropsychological performance at baseline and at follow-up and time effect, group effect and interaction between time and group. SD: standard deviation. The F values refer to those obtained with the repeated-measures general linear model. $F(G \times T)$ : interaction between time and group; $F(G)$ : group effect; $F(T)$ : time effect. 
Supplementary material 4. Local maxima of significant cluster list obtained from model based FEAT analysis. LOP: Left Occpital Pole LOFC: left orbitofrontal cortex; ROFC: right orbitofrontal cortex; APC: anterior paracingulate cortex.

Supplementary material 5. Local maxima of significant cluster list obtained from activation areas that showed decrease activity in IC 1 for PD group over time $(p<0.04)$. RMFG: right middle frontal gyrus; RSPL: right superior parietal lobe; RFP: right frontal pole; ROFC: right orbitofrontal cortex; APC: anterior paracingulate cortex; LSPL: left superior parietal lobe; LOFC: left orbitofrontal cortex; LMTG: left middle temporal gyrus 


\begin{tabular}{|c|c|c|c|c|c|c|}
\hline & \multicolumn{3}{|c|}{$\begin{array}{l}\text { PD patients } \\
\text { Mean (SD) }\end{array}$} & \multicolumn{3}{|l|}{$\begin{array}{l}\text { Controls } \\
\text { Mean (SD) }\end{array}$} \\
\hline & $\begin{array}{l}\text { Initial } \\
\text { sample (n } \\
=24)\end{array}$ & $\begin{array}{l}\text { Final } \\
\text { sample } \\
(\mathbf{n}=17)\end{array}$ & $t / p$ & $\begin{array}{l}\text { Initial sample } \\
(\mathrm{n}=24)\end{array}$ & $\begin{array}{l}\text { Final } \\
\text { sample } \\
(\mathrm{n}=13)\end{array}$ & $t / p$ \\
\hline Age & $56.13(8.5)$ & $56.56(8.6)$ & $\begin{array}{l}-0.17 / \\
0.86\end{array}$ & $57.6(8.9)$ & $\begin{array}{l}57.2 \\
(10.1)\end{array}$ & $\begin{array}{l}0.13 / \\
0.89\end{array}$ \\
\hline $\begin{array}{l}\text { Education } \\
\text { (yrs.) }\end{array}$ & $11.0(5.5)$ & $10.8(4.8)$ & $\begin{array}{l}0.82 / \\
0.94\end{array}$ & $13.00(3.8)$ & $12.3(3.4)$ & $\begin{array}{l}0.55 / \\
0.59\end{array}$ \\
\hline MMSE & $29.6(0.5)$ & $29.5(0.5)$ & $\begin{array}{l}0.60 / \\
0.55\end{array}$ & $29.8(0.4)$ & $29.9(0.3)$ & $\begin{array}{l}-0.75 / \\
0.46\end{array}$ \\
\hline BDI & $6.8(4.8)$ & $7.5(5.3)$ & $\begin{array}{l}-0.49 / \\
0.63\end{array}$ & $4.5(5.1)$ & $3.3(2.4)$ & $\begin{array}{l}0.77 / \\
0.48\end{array}$ \\
\hline NPI & $3.9(7.3)$ & $3.1(5.4)$ & $\begin{array}{l}0.38 / \\
0.71\end{array}$ & $1.4(1.7)$ & $1.1(1.4)$ & $\begin{array}{l}0.54 / \\
0.59\end{array}$ \\
\hline LEDD & $\begin{array}{l}299.6 \\
(321.1)\end{array}$ & $\begin{array}{l}277.7 \\
(308.5)\end{array}$ & $\begin{array}{l}0.22 / \\
0.83\end{array}$ & & & \\
\hline $\begin{array}{l}\text { Disease } \\
\text { duration }\end{array}$ & $3.1(1.6)$ & $2.9(1.4)$ & $\begin{array}{l}0.31 / \\
0.76\end{array}$ & & & \\
\hline Age at onset & $53.1(8.6)$ & $53.7(8.5)$ & $\begin{array}{l}-0.23 / \\
0.82\end{array}$ & & & \\
\hline
\end{tabular}

Supplementary materials 1. 


\begin{tabular}{|l|l|l|l|l|l|l|l|}
\hline & \multicolumn{2}{|l|}{$\begin{array}{l}\text { PD (n=17) } \\
\text { Mean (SD) }\end{array}$} & \multicolumn{2}{l|}{$\begin{array}{l}\text { Controls (n=13) } \\
\text { Mean (SD) }\end{array}$} & $\begin{array}{l}\text { F } \\
(\text { GxT) }\end{array}$ & $\begin{array}{l}\text { F } \\
(G)\end{array}$ & $\begin{array}{l}\text { F } \\
\text { (T) }\end{array}$ \\
\hline & Baseline & Follow-up & Baseline & Follow-up & & & \\
\hline $\begin{array}{l}\text { Phonemic } \\
\text { fluency }\end{array}$ & $\begin{array}{l}13.76 \\
(5.04)\end{array}$ & $\begin{array}{l}15.77 \\
(6.53)\end{array}$ & $\begin{array}{l}16.00 \\
(5.23)\end{array}$ & $\begin{array}{l}17.00 \\
(5.16)\end{array}$ & 0.329 & 0.872 & 2.965 \\
\hline $\begin{array}{l}\text { Semantic } \\
\text { fluency }\end{array}$ & 17.82 & $\begin{array}{l}17.00 \\
(4.80)\end{array}$ & $\begin{array}{l}21.54 \\
(4.58)\end{array}$ & $\begin{array}{l}20.08 \\
(4.82)\end{array}$ & 0.091 & $5.106^{*}$ & 1.163 \\
\hline $\begin{array}{l}\text { Forward digit } \\
\text { span }\end{array}$ & $7.82(1.63)$ & $8.29(1.99)$ & $9.00(1.35)$ & $8.00(2.04)$ & $4.734^{*}$ & 0.613 & 0.613 \\
\hline $\begin{array}{l}\text { Backward } \\
\text { digit span }\end{array}$ & $5.24(1.82)$ & $5.71(1.86)$ & $7.08(1.32)$ & $7.15(2.12)$ & 0.491 & $7.409^{*}$ & 0.949 \\
\hline
\end{tabular}

Supplementary material 3 


\begin{tabular}{|c|c|c|c|c|}
\hline Anatomical region & Voxel & $\mathbf{p}$ & $\mathbf{z}-\mathbf{m a x}$ & $\begin{array}{c}\text { MNI Coordinates } \\
\mathbf{x ~ y ~ z ~}\end{array}$ \\
\hline LOP & 22594 & $3.410^{-39}$ & 8.11 & $-32-94-2$ \\
\hline LOFC & 6787 & $5.8610^{-17}$ & 9.16 & $-3422-8$ \\
\hline ROFC & 6230 & $5.910^{-16}$ & 9.21 & $3620-8$ \\
\hline APC & 3680 & $6.7210^{-11}$ & 8.28 & -63032 \\
\hline
\end{tabular}

Supplementary material 4 


\begin{tabular}{|c|c|c|ccc|}
\hline Anatomical region & Voxels & z-max & \multicolumn{4}{|c|}{ MNI Coordinates } \\
$\mathbf{x}$ y z
\end{tabular}

Supplementary material 5 